\title{
The Variation of Magnetic Properties of Nickel Ferrite by Annealing
}

\author{
H. Moradmard, S. Farjami Shayesteh ${ }^{*}$ \\ Nanostructure Lab, Department of Physics, University of Guilan, Iran
}

Copyright $(2015$ by authors, all rights reserved. Authors agree that this article remains permanently open access under the terms of the Creative Commons Attribution License 4.0 International License

\begin{abstract}
Nickel ferrite nanoparticles were synthesized by Co-precipitation method. The prepared powder was annealed at $500^{\circ} \mathrm{C}, 700^{\circ} \mathrm{C}$ and $900^{\circ} \mathrm{C}$. XRD analysis was used for composition and structure investigation. The detection and confirmation of the chemical bond in spinel ferrites were investigated by Fourier transmission infrared spectra (FTIR). Also the magnetic properties of the samples were measured by using a vibrating sample magnetometer (VSM). The XRD analysis revealed a pure ferrite phase with high crystallinity. The average crystallite size calculated by Sherrer's equation resulted in variation crystallite size from 9 to $21 \mathrm{~nm}$ depending upon the annealing temperature of the samples. FTIR analysis shows the presence of two vibrational bonds between $400-650 \mathrm{~cm}^{-1}$ corresponding to metal-oxygen interaction at tetrahedral and octahedral sites, respectively. Increasing the annealing temperature gave rise to the enhancement of saturation magnetization from 6 to $31 \mathrm{emu} / \mathrm{gr}$ and the variation of coercivity between 2 and $80 \mathrm{Oe}$.
\end{abstract}

Keywords Nickel Ferrite, Co-precipitation, Magnetic Properties

\section{Introduction}

Nanomaterials have received much attention due to their unique properties and various applications which differ from those bulk [1-5]. Among these, the magnetic nanomaterials are more attractive because of their medical, electronic and recording applications. These applications depend on the size, shape, purity and magnetic stability of these materials $[6,7]$. Spinel ferrites are a group of technologically important magnetic materials of current interest which have the general molecular formula $\left(\mathrm{A}^{2+}\right)\left[\mathrm{B}^{3+}\right] \mathrm{O}_{4}{ }^{2-}$ where $\mathrm{A}^{2+}$ and $\mathrm{B}^{3+}$ are divalent and trivalent cations, respectively, occupying the tetrahedral (A) and octahedral (B) interstitial positions of the fcc lattice formed by $\mathrm{O}^{2-}$ ions. Spinel ferrites can be used as antenna rods [8], microwave absorbing materials [9], low and high frequency transformer cores [10], high density information storage materials [11], medical diagnostic tools [12], etc.

Nickel ferrite is the most important class of spinel ferrites. Nickel ferrite has an inverse spinel structure in which the tetrahedral (A-site) are occupied by half of ferric ions and octahedral (B-site) by ferric and nickel ions [7]. Thus the compound can be represented by the formula $\left(\mathrm{Fe}^{3+}\right)$ $\left[\mathrm{Ni}^{2+} \mathrm{Fe}^{3+}\right] \mathrm{O}_{4}{ }^{2-}$. The magnetic structure is generally assumed to be of the Neel collinear type i.e magnetization of the A sublattice is antiparallel of the B sublattice.

Ferrites are usually prepared by various preparation techniques such as sol-gel methods [13], sonochemical technique [14], hydrothermal methods [15], Co-precipitation [16], reverse micelles [17], etc. The preparation technique also plays an important role in surface properties. Among these methods, Co-precipitation method appears to have attracted much attention for preparing ferrite nanoparticles. The advantages of this method are high production rate, very small particle size, low temperature.

\section{Experimental}

\subsection{Synthesis}

Nanoparticles of nickel ferrite were synthesized via Co-precipitation method. The starting materials were iron chloride hexahydrate $\left(\mathrm{FeCl}_{3} \cdot 6 \mathrm{H}_{2} \mathrm{O}\right)$, nickel chloride hexahydrate $\left(\mathrm{NiCl}_{2} \cdot 6 \mathrm{H}_{2} \mathrm{O}\right)$ and sodium hydroxide $(\mathrm{NaOH})$ was used as the precipitating agent. All the reagents used in the experiments were supplied by Chem-Lab. We have used 0.1 and $0.2 \mathrm{M}$ solution from nickel and iron chloride, respectively. Salts dissolved in $50 \mathrm{ml}$ double distilled water. Double distilled de-ionized water was used as the solvent to minimize the impurities in the final product. The mixed solution was added to Sodium hydroxide solution (2 M) drop wise till $\mathrm{PH}$ received close to 13 . The reaction was carried out at $80^{\circ} \mathrm{C}$ for $2 \mathrm{~h}$ with vigorous mixing. The precipitated product was centrifuged at $3200 \mathrm{rpm}$ and the products were washed with deionized water for several times. Then 
powders dried at $60^{\circ} \mathrm{C}$ for $24 \mathrm{~h}$. The obtained powders were annealed at 500 and 700 and $900^{\circ} \mathrm{C}$ for $2 \mathrm{~h}$ by using a furnace.

\subsection{Characterization}

$\mathrm{X}$-ray diffractometer (XRD) patterns were obtained using a PW 1800 (Philips, Netherland) with $\mathrm{Cu}-\mathrm{K}_{\alpha}$ radiation $(\lambda=$ $0.154 \mathrm{~nm})$ in the $2 \theta$ range of $20-80^{\circ}$. Fourier transform infrared spectroscopy (FTIR, Nicolet Magna, IR560, USA) studies were carried out on the samples to examine the compositional characteristics. Magnetic properties of samples were obtained using Vibrating Sample Magnetometer (VSM, Meghnatis Daghigh Kavir Co, Iran).

\section{Results and Discussion}

Figure.1 shows the XRD pattern of as-prepared and annealed nickel ferrite nanoparticles. Formation of single phase cubic spinel $\mathrm{NiFe}_{2} \mathrm{O}_{4}$ nanoparticles in all the samples was confirmed. The average crystallite size was calculated by using the Scherrer's formula [18]:

$$
D=\frac{0.9 \lambda}{\beta \cos \theta}
$$

Where $\lambda$ is the X-ray wavelength $(\lambda=0.154 \mathrm{~nm}$ for $\left.\mathrm{CuK}_{\alpha}\right), \beta$ is the full width at half maximum, $\theta$ is the Bragg angle. The measurement was performed in the step range of 0.02 degrees from $20^{\circ}$ to $80^{\circ}$. The crystallite sizes of the samples are summarized in table 1 .

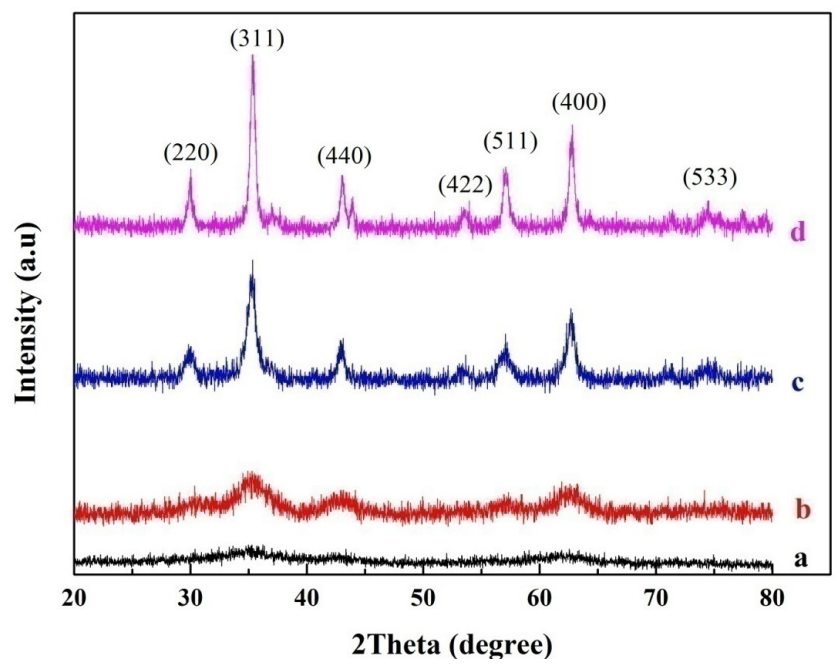

Figure 1. X-ray diffraction patterns for $\mathrm{NiFe}_{2} \mathrm{O}_{4}$ nanoparticles (a) as-prepared and annealed at (b) $500^{\circ} \mathrm{C}$, (c) $700^{\circ} \mathrm{C}$ and (d) $900^{\circ} \mathrm{C}$.
Figure 1 (a) reveals evidence of an amorphous phase. According to the Figure 1, it is clear that by increasing in annealing temperature the reflection peaks become sharper and narrower. X-Ray pattern illustrates the evolution of crystallinity of the stoichiometric $\mathrm{Ni}$-ferrite with increasing annealing temperature. Increasing the annealing temperature improves the samples crystallization and thus increases the grain size. So the grains become larger as the annealing temperature increases. The nanometer size grains possess a large overall surface area which means that their total surface energy is also high. However the energy can be minimized when the annealing process was carried out, thus allowing the grains to agglomerate. Further increasing the annealing temperature reduced the surface energy of the grains and thus allowed them to become more agglomerate [18]. The diffraction peaks correspond to (220), (311), (400), (422), (511) and (440) planes which related to spinel structure (JCPDS, Card No.10-325). The annealed samples at 500, 700 , and $900^{\circ} \mathrm{C}$ show average crystallite sizes of 9,14 and 21 $\mathrm{nm}$, respectively. Increase in the annealing temperature yields a growth of the larger grain size of $\mathrm{Ni}$ ferrite particles. It was reported that annealing process generally decreases lattice defects and strains. However, it can also cause coalescence of smaller grains that result in increasing the average grain size of the nanoparticles [19]. The observed increase in average crystallite size with annealing temperature is most likely due to the fact that higher annealing temperature and time enhances the coalescence process resulting in an increase in the grain size. Thus, it appears that particle size may be controlled either by varying the annealing temperature of the sample or the annealing time during the synthesis process [20].

Lattice constant was calculated by using the following equation [18]

$$
a=d_{h k l} \sqrt{h^{2}+k^{2}+l^{2}}
$$

Where $d_{h k l}$ is the inter plane spacing; $h, k$, and 1 are the Miller indices of the crystal planes. The calculated parameters for all samples are given in table 1 , which are in close agreement with standard data (8.34 $\AA$ ) [21].

The X-ray density of $\mathrm{NiFe}_{2} \mathrm{O}_{4}$ nanoparticles is calculated using the formula [18]

$$
P_{x}=\frac{8 M}{N a^{3}}
$$

and it is given in table 1 . Where $M$ is the molecular weight of the sample, $N$ the Avogadro's number (per mol) and $a$ the lattice constant. 
Table 1. Average crystallite sizes, lattice parameters and X-Ray density calculated from XRD results and magnetic properties measured from VSM technique at room temperature for nickel ferrite nanoparticles annealed at 500, 700 and $900{ }^{\circ} \mathrm{C}$.

\begin{tabular}{|c|c|c|c|c|c|}
\hline $\begin{array}{c}\text { Annealing } \\
\text { Temperature }\left({ }^{\circ} \mathrm{C}\right)\end{array}$ & $\begin{array}{c}\text { Average } \\
\text { crystallite size } \\
(\mathrm{nm})\end{array}$ & $\begin{array}{c}\text { Lattice } \\
\text { constant } \\
\left({ }^{\circ} \mathrm{A}\right)\end{array}$ & $\begin{array}{c}\text { X-Ray density } \\
\left(\mathrm{kg} / \mathrm{cm}^{3}\right)\end{array}$ & $\begin{array}{c}\text { Saturation Magnetization } \\
M_{s}(\mathrm{emu} / \mathrm{gr})\end{array}$ & $\begin{array}{c}\text { Coercivity } \\
H_{c}(\mathrm{O} e)\end{array}$ \\
\hline 500 & 9 & 8.48 & 5.10 & 6 & 21 \\
\hline 700 & 14 & 8.41 & 5.23 & 31 & 8 \\
\hline 900 & 21 & 8.40 & 5.25 & 80 \\
\hline
\end{tabular}

FESEM image was used to characterize the morphology of annealed nickel ferrite at $900^{\circ} \mathrm{C}$ which was shown in Figure 2. Micrograph confirms the formation of spherical particles for $\mathrm{NiFe}_{2} \mathrm{O}_{4}$.

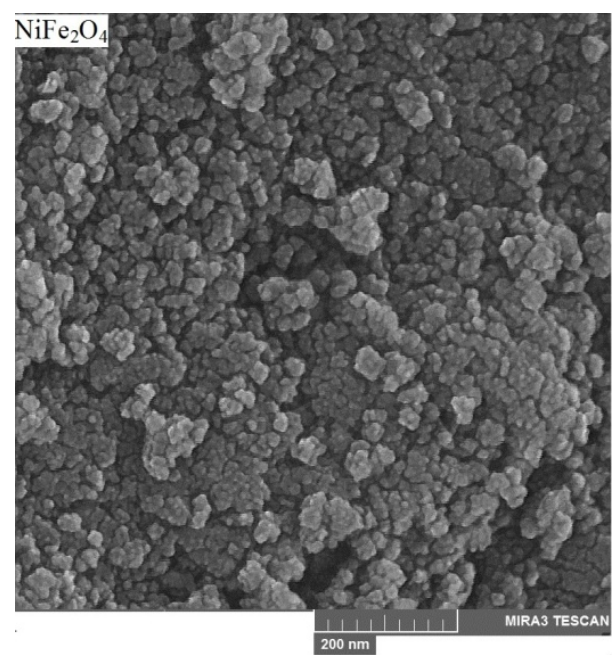

Figure 2. FESEM image of $\mathrm{NiFe}_{2} \mathrm{O}_{4}$ nanoparticles, which annealed at $900^{\circ} \mathrm{C}$.

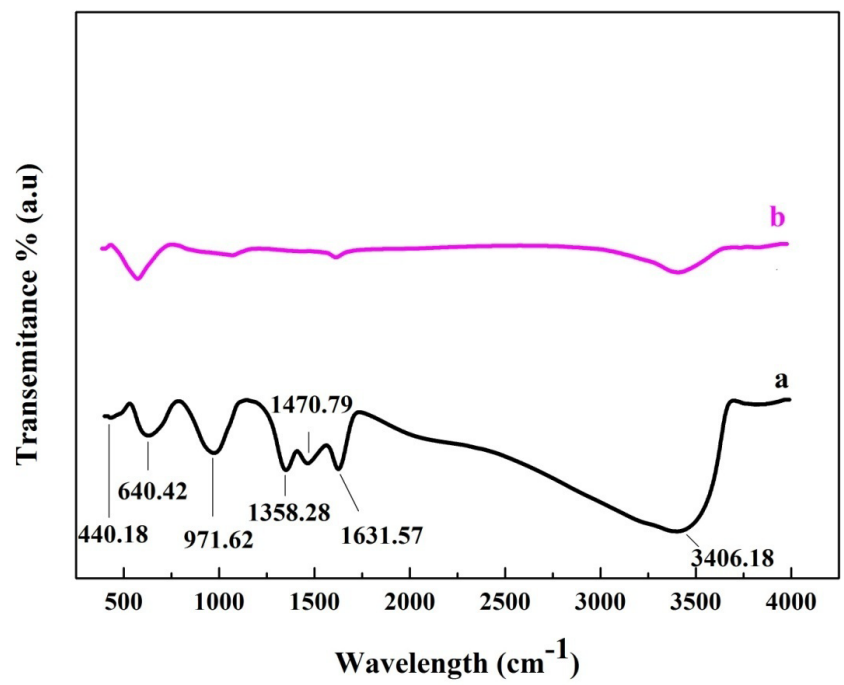

Figure 3. FTIR spectra of a) as-preparedb) annealed at $900^{\circ} \mathrm{C} \mathrm{NiFe}_{2} \mathrm{O}_{4}$ nanoparticles.

The FTIR spectrum of as-prepared Nickel ferrite and annealed at $900^{\circ} \mathrm{C}$ are presented in Figure 3. It shows the two principle absorption bands in the range of $400-650 \mathrm{~cm}^{-1}$ in which the first band is around $440 \mathrm{~cm}^{-1}$ and the second band is around $636 \mathrm{~cm}^{-1}$, attributed to the long bond length of oxygen-metal ions in the octahedral sites and the shorter bond length of oxygen-metal ions in the tetrahedral sites in the spinel structure, respectively [22].

The band around $1600 \mathrm{~cm}^{-1}$ corresponds to bending mode of $\mathrm{H}_{2} \mathrm{O}$ molecules [23]. The absorption broadband at 3400 $\mathrm{cm}^{-1}$ represents a stretching mode of $\mathrm{H}_{2} \mathrm{O}$ molecules and $\mathrm{OH}$ groups [24]. It is clear that after annealing, $\mathrm{O}-\mathrm{H}$ bonds were vanishing in annealed sample.

The magnetic properties of as-prepared and annealed samples have been characterized at room temperature using a vibrating sample magnetometer (VSM) and the magnetic hysteresis loops are shown in Figure 4. These curves are typical for a soft magnetic material and indicate hysteresis ferromagnetism. It can be seen from Figure 4 that the increase in annealing temperature resulted in an increase in both the saturation magnetization $\left(\mathrm{M}_{\mathrm{s}}\right)$ and coercivity $\left(\mathrm{H}_{\mathrm{c}}\right)$ of samples. It is found that the increase tendency of $\mathrm{M}_{\mathrm{s}}$ and $\mathrm{H}_{\mathrm{c}}$ are consistent with the improvement of crystallinity of the samples. The saturation magnetization increases rapidly from 2 to $31 \mathrm{emu} / \mathrm{g}$ with increasing annealing temperature, as shown in Figure 4. However, the coercivity increases with annealing temperature to $80 \mathrm{Oe}$. In case of nickel ferrite any configurations of $\mathrm{Ni}^{2+}$ and $\mathrm{Fe}^{3+}$ ions in both octahedral and tetrahedral sites tend to increase the net magnetization per formula unit [25].

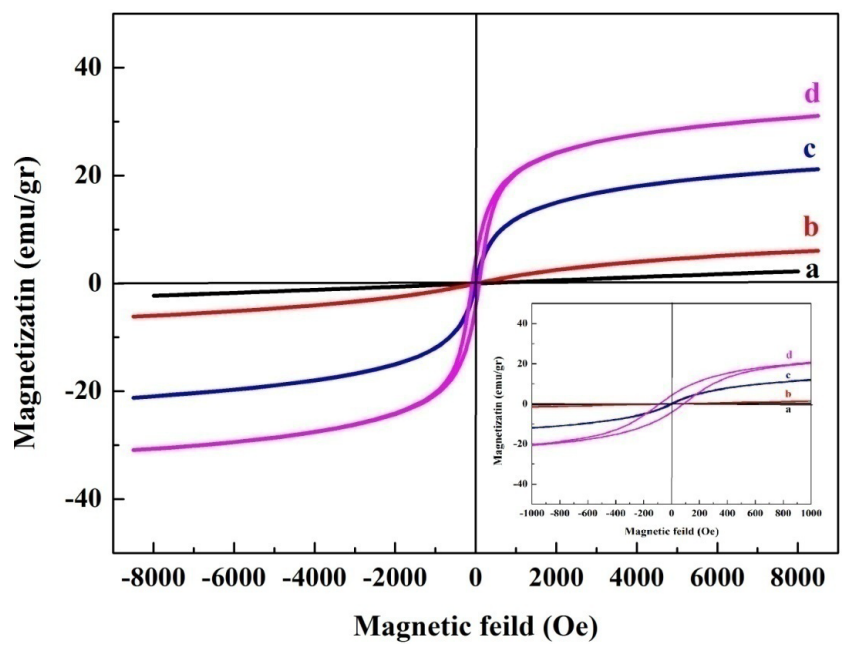

Figure 4. Magnetization curves as a function of magnetic field for $\mathrm{NiFe}_{2} \mathrm{O}_{4}$ nanoparticles (a) as prepared and annealed at (b) $500^{\circ} \mathrm{C}$, (c) $700^{\circ} \mathrm{C}$, and (d) $900^{\circ} \mathrm{C}$. 
These changes might be due to the variation in size, distribution of ions, canting effect and shape of nanoparticles. The dependence of the magnetization and magnetic momentom to the grain size is explained on the basis of changes in the exchange interaction between tetrahedral and octahedral sub-lattices [26]. Four factors, namely super-exchange interaction, magneto crystalline anisotropy, canting effect and dipolar interactions between the projected moments on the surface of the nanoparticles should be considered $[27,26]$. On the other hand, the variation of $\mathrm{H}_{\mathrm{c}}$ with particle size can be explained on the basis of the domain structure, critical diameter and anisotropy of the crystal [2830].

\section{Conclusions}

The XRD studies of the Co-precipitation synthesized $\mathrm{NiFe}_{2} \mathrm{O}_{4}$ showed that samples have the spinel structure and the X-ray data agreed well with the reported data [31]. It was observed that the grain size changes with increasing of annealing temperature. Crystallinity of samples and the average crystallite size was increased by the annealing process. Magnetic studies showed that the saturation magnetization and coercivity of the nanosized $\mathrm{NiFe}_{2} \mathrm{O}_{4}$ increased as the grain size increased. The magnetization of the annealed samples increased with the annealing temperature. It is apparent that enhancement of annealing temperature cause to increase of crystallite size and magnetization of nanopowders.

\section{REFERENCES}

[1] G. Mandal, T. Ganguly, "Applications of nanomaterials in the different fields of photosciences"; Indian J. Phys. 85 (2011) 1229-1245.

[2] S. Mitra, A. Mandal, S. Banerjee, A. Datta, S. Bhattacharya, A. Bose, D. Chakravorty,"Template based growth of nanoscaled films: a brief review”; Indian J. Phys. 85 (2011) 649-666.

[3] S. Karan, D. Dutta Majumder, A. Goswami, "A mathematical formalism of self assembly for design and fabrication of nanostructured materials: a new paradigm for nanotechnology"; Indian J. Phys. 86(2012) 667-676.

[4] J. Bhadra, D. Sarkar, "Field effect transistor fabricated from polyaniline-polyvinyl alcohol nanocomposite"; Indian J. Phys. 84 (2010) 693-697.

[5] M. R Vaezi, S. K. Shendy, T. Ebadzadeh, "Synthesis of $\mathrm{TiO} 2 / \mathrm{SnO} 2$ core shell nanocomposite by chemical route and its gas sensing properties"; Indian J. Phys. 86 (2012) 9-13.

[6] K. Maaz, S. Karim, A. Mumtaz, K. Hasanain, "Synthesis and magnetic characterization of nickel ferrite nanoparticles prepared by Co-precipitation route"; J. Magn. Magn. Mater. 321 (2009) 1838-1842.

[7] A. Ceylanab, S. Ozcan, C. Ni, SI. Shah, "Solid state reaction synthesis of $\mathrm{NiFe}_{2} \mathrm{O}_{4}$ nanoparticles"; J. Magn. Magn. Mater. 320 (2008) 857-863.
[8] M. Sugimoto,"ChemInform Abstract: The Past, Present, and Future of Ferrites"; J. Am. Ceram. Soc. 82 (1999) 269-280.

[9] D. Zhao, Q. Lv, Z. Shen, "Fabrication and microwave absorbing properties of $\mathrm{Ni}-\mathrm{Zn}$ spinel ferrites"; J. Alloy. Compd. 480 (2009) 634-638.

[10] B.P. Rao, K. H. Rao, "Design aspects in processing of Ni-Zn ferrites for high frequency applications"; J. Mater. Sci. Lett 2 (2003) 1607-1608.

[11] M.M. Bucko, K. Haberko, "Hydrothermal synthesis of nickel ferrite powders, their properties and sintering"; J. Eur. Ceram. Soc. 27 (2007) 723-727.

[12] D.H. Kim, H. Zeng, T.C. Ng, C.S. Brazel, " $\mathrm{T}_{1}$ and $\mathrm{T}_{2}$ relaxivities of succimer-coated $\mathrm{MFe}_{2}{ }^{3+} \mathrm{O}_{4}\left(\mathrm{M}=\mathrm{Mn}^{2+}, \mathrm{Fe}^{2+}\right.$ and $\mathrm{Co}^{2+}$ ) inverse spinel ferrites for potential use as phase-contrast agents in medical MRI"; J. Magn. Magn.Mater. 321(2009) 3899 .

[13] D.H. Chen, X.R. He, "Synthesis of nickel ferrite nanoparticles by sol-gel method"; Mater. Res. Bull. 36 (2001) 1369-1377.

[14] K.V.P.M. Shafi, Y. Koltypin, A. Gedanken, R. Prozorov, J. Balogh, J. Lendvai, et al, "Sonochemical Preparation of Nanosized Amorphous $\mathrm{NiFe}_{2} \mathrm{O}_{4}$ Particles"; J. Phys. Chem. B 101 (1997) 6409-6414.

[15] H. Li, H.Z. Wu, G.X. Xiao, "Effects of synthetic conditions on particle size and magnetic properties of $\mathrm{NiFe}_{2} \mathrm{O} 4$ "; Powder Technol. 198 (2010) 157-166.

[16] M.S. Niasari, F. Davar, T. Mahmoudi, "A simple route to synthesize nanocrystalline nickel ferrite $\left(\mathrm{NiFe}_{2} \mathrm{O}_{4}\right)$ in the presence of octanoic acid as a surfactant"; Polyhedron 28 (2009) 1455-1458.

[17] A. Kale, S. Gubbala, R.D.K. Misra, "Magnetic behavior of nanocrystalline nickel ferrite synthesized by the reverse micelle technique"; J. Magn. Magn. Mater. 277 (2004) 350358.

[18] A. Khorsand Zak, W. H. Abd Majid, M. E. Abrishami, R. Yousefi, "Synthesis and characterization of a narrow size distribution of zinc oxide nanoparticles"; Solid State Sci. (2011) 13251-256.

[19] N. B. Ibrahim, Ftema W. Aldbea, and Mustaffa Hj Abdullah, "Effects of Annealing Temperature on Structure and Magnetic Properties of $\mathrm{Tb}_{x} \mathrm{Y}_{3-x} \mathrm{~F}_{5} \mathrm{O}_{12}(x=0.2$ and 0.4$)$ Thin Films"; Journal of Nanomaterials, 2012 (2012), 154192-154197

[20] T. P. Raming, A. J. A. Winnubst, C. M. van Kats, P. Philipse, "The Synthesis and Magnetic Properties of Nanosized Hematite $\left(\alpha-\mathrm{Fe}_{2} \mathrm{O}_{3}\right)$ Particles"; J. Colloid Interface Sci. 249 (2002) 346-350.

[21] J. Smit, H.P. Wijn,"Ferrites";(The Netherlands: Philips Technical Library) (1959) 137-141.

[22] Y. Ahn, E.J. Choi, S. Kim, H.N. Ok, "Magnetization and Mössbauer study of cobalt ferrite particles from nanophase cobalt iron carbonate"; Mater. Lett. 50 (2001) 47-52.

[23] L. Nasdala, A, Beran, E. Libowitzky, D. Wolf, "The Incorporation of hydroxsyl groups and molecular water in natural zircon $\left(\mathrm{ZrSiO}_{4}\right)$ "; American Journal of Science, 301 (2001) 831-857. 
[24] G.S.R. Krishnamurti, P.M. Huang, "Influence of citrate on the kinetics of $\mathrm{Fe}$ (II) oxidation and the formation of iron oxyhydroxides"; Clays Clay Miner. 39 (1991) 28-34.

[25] J.G.S. Duque, E.A. Souza, C.T.Meneses, L. Kubota, "Magnetic properties of $\mathrm{NiFe}_{2} \mathrm{O}_{4}$ nanoparticles produced by a new chemical method"; Physica B 398 (2007) 287-290.

[26] R.H. Kodama, A.E. Berkowitz, "Atomic-scale magnetic modeling of oxide nanoparticles"; Phys. Rev. B 59 (1999) 6321-6325.

[27] R.H. Kodama, A.E. Berkowitz, E.J. McNiff Jr., S. Foner, "Surface spin disorder in $\mathrm{NiFe}_{2} \mathrm{O}_{4}$ nanoparticles"; Phys. Rev. Lett. 77 (1996) 394-400.
[28] B.D. Cullity, "Introduction to Magnetic Materials"; Addison-Wesley Publishing (1972).

[29] S. Chikazumi, "Physics of Magnetism"; Wiley, New York, (1959).

[30] M. Georgea,A. Mary John, S.S.Naira, P.A. Joy, M.R. Anantharaman, "Finite size effects on the structural and magnetic properties of sol-gel synthesized $\mathrm{NiFe}_{2} \mathrm{O}_{4}$ powders"; J. Magn. Magn. Mater. 302 (2006) 190-195.

[31] Y. Shi, J. Ding, X. Liu, J. Wan, " $\mathrm{NiFe}_{2} \mathrm{O}_{4}$ ultrafine particles prepared by Co-precipitation/mechanical alloying"; J. Mag. Mag. Mater 205 (1999) 249-254. 